CRYSTALLOGRAPHIC COMMUNICATIONS

ISSN 2056-9890

Received 11 January 2016

Accepted 18 January 2016

Edited by M. Weil, Vienna University of Technology, Austria

Keywords: crystal structure; azamacrocyclic ligand; isonicotinic acid; $\pi-\pi$ interactions; synchrotron data

CCDC reference: 1447865

Supporting information: this article has

supporting information at journals.iucr.org/e

\section{Crystal structure of trans-(1,8-dibutyl- $1,3,6,8,10,13$-hexaazacyclotetradecane- $\kappa^{4} N^{3}, N^{6}, N^{10}, N^{13}$ )bis(isonicotinato- $\kappa O$ ) nickel(II) determined from synchrotron data}

\author{
Jong Won Shin, Dae-Woong Kim and Dohyun Moon*
}

Beamline Department, Pohang Accelerator Laboratory 80, Jigokro-127-beongil, Nam-Gu Pohang, Gyeongbuk 37673 , Republic of Korea. ${ }^{*}$ Correspondence e-mail: dmoon@postech.ac.kr

The title compound, $\left[\mathrm{Ni}\left(\mathrm{C}_{6} \mathrm{H}_{4} \mathrm{NO}_{2}\right)_{2}\left(\mathrm{C}_{16} \mathrm{H}_{38} \mathrm{~N}_{6}\right)\right]$, was prepared through selfassembly of a nickel(II) azamacrocyclic complex with isonicotinic acid. The $\mathrm{Ni}^{\mathrm{II}}$ atom is located on an inversion center and exhibits a distorted octahedral $\mathrm{N}_{4} \mathrm{O}_{2}$ coordination environment, with the four secondary $\mathrm{N}$ atoms of the azamacrocyclic ligand in the equatorial plane [average $\mathrm{Ni}-\mathrm{N}_{\mathrm{eq}}=2.064$ (11) $\AA$ ] and two $\mathrm{O}$ atoms of monodentate isonicotinate anions in axial positions $\left[\mathrm{Ni}-\mathrm{O}_{\mathrm{ax}}=\right.$ 2.137 (1) $\AA$ ]. Intramolecular $\mathrm{N}-\mathrm{H}$. . O hydrogen bonds between one of the secondary amine $\mathrm{N}$ atoms of the azamacrocyclic ligand and the noncoordinating carboxylate $\mathrm{O}$ atom of the anion stabilize the molecular structure. Intermolecular $\mathrm{N}-\mathrm{H} \cdots \mathrm{N}$ hydrogen bonds, as well as $\pi-\pi$ interactions between neighbouring pyridine rings, give rise to the formations of supramolecular ribbons extending parallel to [001].

\section{Chemical context}

The molecular design and synthesis of coordination polymers with macrocyclic ligands have attracted considerable attention because of their potential applications in chemistry, environmental chemistry, and materials science (Churchard et al., 2010; Lehn, 2015). To obtain specific molecular compounds through assembly of supramolecular building blocks with properties such as guest recognition or catalytic effects, macrocyclic complexes involving vacant sites in an axial position are good candidates. Moreover, these complexes can also be easily derivatized by carboxylic acid moieties, such as 1,3,5-BTC (1,3,5-benzenetricarboxylic acid), 2,7-NDC (2,7naphthalenedicarboxylic acid) or 1,3,5-CTC (1,3,5-cyclohexanetricarboxylic acid), forming interesting coordination compounds with supramolecular structures ranging from chains to networks (Min \& Suh, 2001; Shin et al., 2016b). For example, $\left[\mathrm{Ni}\left(L^{R},{ }^{R}\right)\right]_{3}\left[\mathrm{BTC}{ }^{3-}\right]_{2} \cdot 12 \mathrm{H}_{2} \mathrm{O} \cdot \mathrm{CH}_{3} \mathrm{CN} \quad\left(L^{R R},{ }^{2}=1,8-\right.$ bis $[(R)$ - $\alpha$-methylbenzyl $]-1,3,6,8,10,13$-hexaazacyclotetradecane) displays a two-dimensional supramolecular network structure and exhibits a selective chiral recognition for racemic material (Ryoo et al., 2010). Isonicotinic acid as another building unit can easily bind or interact with transition metal ions through its possible bridging or coordination modes associated with the carboxylic group and pyridine moieties, respectively, thus allowing the assembly of compounds with supramolecular structures or the formation of heterometallic complexes (Xie et al., 2014).

Here, we report on the synthesis and crystal structure of an $\mathrm{Ni}^{\mathrm{II}}$ azamacrocyclic complex including isonicotinate anions, $\left[\mathrm{Ni}\left(\mathrm{C}_{6} \mathrm{H}_{4} \mathrm{NO}_{2}\right)_{2}\left(\mathrm{C}_{16} \mathrm{H}_{38} \mathrm{~N}_{6}\right)\right]$, (I). 


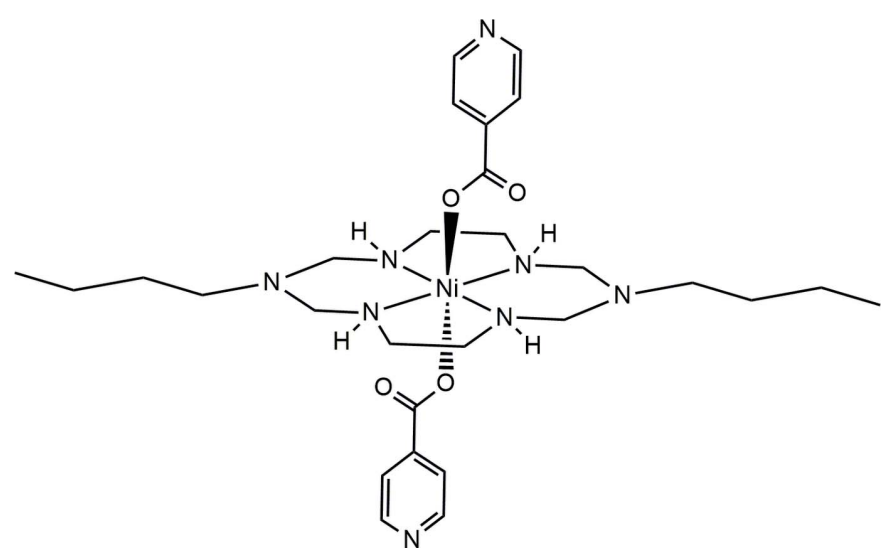

\section{Structural commentary}

Compound (I) is isotypic with its copper(II) analogue (Shin et al., 2015). The nickel(II) atom is located on an inversion center. The coordination environment around the nickel(II) atom is distorted octahedral with the four secondary amine $\mathrm{N}$ atoms of the azamacrocyclic ligand in the equatorial plane and two $\mathrm{O}$ atoms of two monodentate isonicotinate anions in axial positions (Fig. 1). The average $\mathrm{Ni}-\mathrm{N}_{\mathrm{eq}}$ bond lengths is 2.064 (11) $\AA$ and the $\mathrm{Ni}-\mathrm{O}_{\mathrm{ax}}$ bond length is 2.137 (1) $\AA$. The longer axial bonds can be attributed to a ring contraction of the azamacrocyclic ligand (Melson, 1979). The six-membered $\mathrm{NiC}_{2} \mathrm{~N}_{3}$ ring (Ni1-N1-C2-N3-C3-N2) adopts the expected

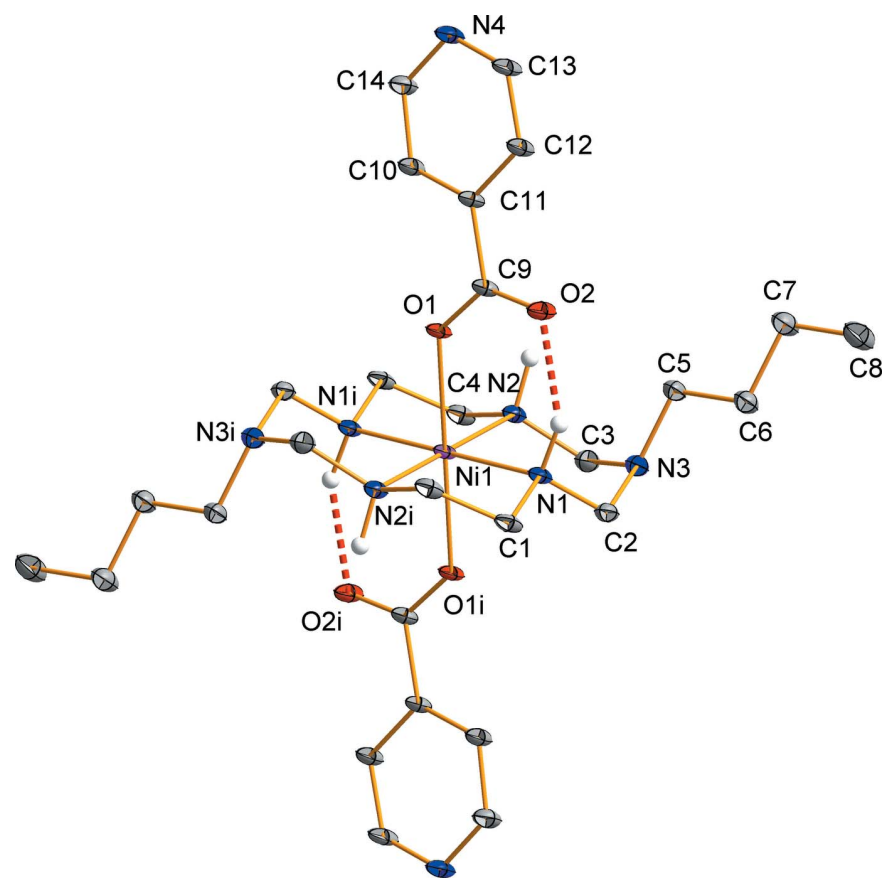

Figure 1

View of the molecular structure of the title compound, showing the atomlabelling scheme, with displacement ellipsoids drawn at the $30 \%$ probability level. $\mathrm{H}$ atoms bonded to $\mathrm{C}$ atoms have been omitted for clarity. Intramolecular $\mathrm{N}-\mathrm{H} \cdots \mathrm{O}$ hydrogen bonds are shown as red dashed lines. [Symmetry code: (i) $-x+1,-y+1,-z+1$.]
Table 1

Hydrogen-bond geometry $\left(\AA,^{\circ}\right)$.

\begin{tabular}{lllll}
\hline$D-\mathrm{H} \cdots A$ & $D-\mathrm{H}$ & $\mathrm{H} \cdots A$ & $D \cdots A$ & $D-\mathrm{H} \cdots A$ \\
\hline $\mathrm{N} 1-\mathrm{H} 1 \cdots \mathrm{O} 2$ & 1.00 & 1.98 & $2.892(2)$ & 150 \\
$\mathrm{~N} 2-\mathrm{H} 2 \cdots \mathrm{N} 4^{\mathrm{i}}$ & 1.00 & 2.23 & $3.143(2)$ & 151 \\
\hline
\end{tabular}

Symmetry code: (i) $-x+1,-y+1,-z$.

chair conformation, whereas the five-membered $\mathrm{NiC}_{2} \mathrm{~N}_{2}$ ring (Ni1-N1-C1-C4-N2) has a gauche conformation (Min \& Suh, 2001). Since the carboxylate group is fully delocalized, the two $\mathrm{C}-\mathrm{O}$ bonds and the bond angle $(\mathrm{O} 1-\mathrm{C} 9-\mathrm{O} 2)$ are $1.267(2)$, 1.248 (2) $\AA$ and $126.9(2)^{\circ}$, respectively. The bond angles around the nickel(II) atom are in the normal range for an octahedral complex. Intramolecular $\mathrm{N}-\mathrm{H} \cdots \mathrm{O}$ hydrogen bonds between one of the secondary amine groups of the azamacrocyclic ligand and the non-coordinating carboxylate $\mathrm{O}$ atom of the isonicotinate anion form six-membered rings and stabilize the molecular structure (Fig. 1, Table 1).

\section{Supramolecular features}

The N4 atom of the isonicotinate anion forms an intermolecular hydrogen bond with an adjacent secondary amine group of the azamacrocyclic ligand (Fig. 2, Table 1) (Steed \& Atwood, 2009). In addition, parallel pyridine rings (Hunter \& Sanders, 1990) of the isonicotinate anions participate in $\pi-\pi$ interactions with a centroid-to-centroid distance of 3.741 (1) $\AA$ and an interplanar separation of 3.547 (1) $\AA$. The interplay between hydrogen bonds and $\pi-\pi$ interactions give rise to the formation of supramolecular ribbons extending parallel to [001].

\section{Database survey}

A search of the Cambridge Structural Database (Version 5.36, May 2014 with 3 updates; Groom \& Allen, 2014) reveals two

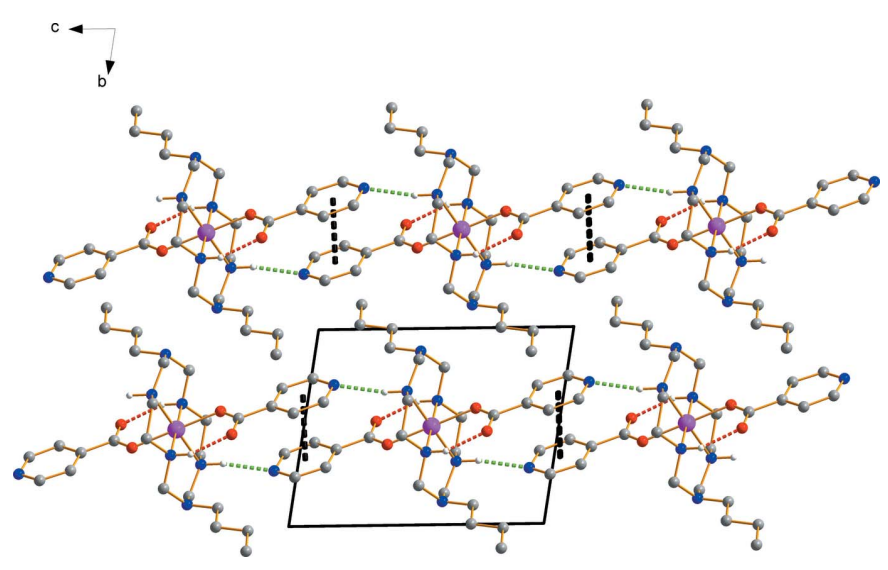

Figure 2

View of the crystal packing of the title compound, showing hydrogen bonds and $\pi-\pi$ interactions (red: intramolecular $\mathrm{N}-\mathrm{H} \cdots \mathrm{O}$ hydrogen bonds, green: intermolecular $\mathrm{N}-\mathrm{H} \cdots \mathrm{N}$ hydrogen bonds, black: $\pi-\pi$ interactions). 
complexes with the same nickel(II) azamacrocyclic building block (Kim et al., 2015a,b) for which synthesis, FT-IR spectroscopic data and the crystal structure have been reported.

\section{Synthesis and crystallization}

The starting complex, $\left[\mathrm{Ni}\left(\mathrm{C}_{16} \mathrm{H}_{38} \mathrm{~N}_{6}\right)\left(\mathrm{ClO}_{4}\right)_{2}\right]$, was prepared in a slightly modified procedure with respect to the reported method (Kim et al., 2015b). To an acetonitrile solution $(14 \mathrm{~mL})$ of $\left[\mathrm{Ni}\left(\mathrm{C}_{16} \mathrm{H}_{38} \mathrm{~N}_{6}\right)\left(\mathrm{ClO}_{4}\right)_{2}\right](0.298 \mathrm{~g}, 0.52 \mathrm{mmol})$ was slowly added an acetonitrile solution $(8 \mathrm{~mL})$ containing isonicotinic acid $(0.128 \mathrm{~g}, 1.04 \mathrm{mmol})$ and excess triethylamine $(0.12 \mathrm{~g}, 1.20 \mathrm{mmol})$ at room temperature. The purple precipitate was filtered off, washed with acetonitrile and diethyl ether, and dried in air. Single crystals of compound (I) were obtained by layering of the acetonitrile solution of isonicotinic acid on the acetonitrile solution of $\left[\mathrm{Ni}\left(\mathrm{C}_{16} \mathrm{H}_{38} \mathrm{~N}_{6}\right)\left(\mathrm{ClO}_{4}\right)_{2}\right]$ for several days. Yield: $0.167 \mathrm{~g}(52 \%)$. FT-IR (ATR, $\left.\mathrm{cm}^{-1}\right)$ : 3145 , 3075, 2951, 2920, 1571, 1457, 1351, 1272, 1014, 915.

Safety note: Although we have experienced no problems with the compounds reported in this study, perchlorate salts of metal complexes are often explosive and should be handled with great caution.

\section{Refinement}

Crystal data, data collection and structure refinement details are summarized in Table 2. All $\mathrm{H}$ atoms were placed in geometrically idealized positions and constrained to ride on their parent atoms, with $\mathrm{C}-\mathrm{H}$ distances of 0.95 (ring $\mathrm{H}$ atoms) or $0.98-0.99 \AA$ (open-chain $\mathrm{H}$ atoms), and an $\mathrm{N}-\mathrm{H}$ distance of $1.0 \AA$, with $U_{\text {iso }}(\mathrm{H})$ values of 1.2 or $1.5 U_{\text {eq }}$ of the parent atoms.

\section{Acknowledgements}

This work was supported by Basic Science Research Program through the National Research Foundation of Korea (NRF) funded by the Ministry of Education, Science and Technology (NRF-2014R1A1A2058815) and supported by the Institute for Basic Science (IBS-R007-D1-2016-a01). The X-ray crystallography BL2D-SMC beamline and FT-IR experiment at the PLS-II are supported in part by MSIP and POSTECH.

\section{References}

Churchard, A. J., Cyranski, M. K., Dobrzycki, Ł., Budzianowski, A. \& Grochala, W. (2010). Energ. Environ. Sci. 3, 1973-1978.

Groom, C. R. \& Allen, F. H. (2014). Angew. Chem. Int. Ed. 53, 662671.

Hunter, C. A. \& Sanders, K. M. (1990). J. Am. Chem. Soc. 112, 55255534.
Table 2

Experimental details.

\begin{tabular}{ll}
\hline Crystal data & \\
Chemical formula & {$\left[\mathrm{Ni}\left(\mathrm{C}_{6} \mathrm{H}_{4} \mathrm{NO}_{2}\right)_{2}\left(\mathrm{C}_{16} \mathrm{H}_{38} \mathrm{~N}_{6}\right)\right]$} \\
$M_{\mathrm{r}}$ & 617.44 \\
Crystal system, space group & Triclinic, $P \overline{1}$ \\
Temperature $(\mathrm{K})$ & 100 \\
$a, b, c(\AA)$ & $8.0630(16), 8.5110(17), 10.927(2)$ \\
$\alpha, \beta \gamma^{\prime}\left({ }^{\circ}\right)$ & $80.52(3), 88.26(3), 86.44(3)$ \\
$V\left(\AA^{3}\right)$ & $738.0(3)$ \\
$Z$ & 1 \\
Radiation type & Synchrotron, $\lambda=0.62998 \AA$ \\
$\mu\left(\mathrm{mm}^{-1}\right)$ & 0.51 \\
Crystal size $(\mathrm{mm})$ & $0.01 \times 0.004 \times 0.004$ \\
& \\
Data collection & $\mathrm{ADSC} \mathrm{Q} 210 \mathrm{CCD}$ area detector \\
Diffractometer & Empirical $(H K L-3000 S M S C A L E-$ \\
Absorption correction & $P A C K ;$ Otwinowski \& Minor, \\
& $1997)$ \\
$T_{\text {min }}, T_{\max }$ & $0.995,0.998$ \\
No. of measured, independent and & $7634,3879,3326$ \\
$\quad$ observed $[I>2 \sigma(I)]$ reflections & \\
$R_{\text {int }}$ & 0.023 \\
$(\text { sin } \theta / \lambda)_{\text {max }}\left(\AA^{-1}\right)$ & 0.696 \\
& \\
Refinement & \\
$R\left[F^{2}>2 \sigma\left(F^{2}\right)\right], w R\left(F^{2}\right), S$ & $0.040,0.110,1.04$ \\
No. of reflections & 3879 \\
No. of parameters & 188 \\
$\mathrm{H}$-atom treatment & $\mathrm{H}$-atom parameters constrained \\
$\Delta \rho_{\text {max }}, \Delta \rho_{\text {min }}\left(\mathrm{e} \AA^{-3}\right)$ & $1.12,-0.95$ \\
&
\end{tabular}

Computer programs: PAL BL2D-SMDC (Shin et al., 2016a), HKL-3000SM (Otwinowski \& Minor, 1997), SHELXT (Sheldrick, 2015a), SHELXL2014 (Sheldrick, 2015b), DIAMOND (Putz \& Brandenburg, 2014) and publCIF (Westrip, 2010).

Kim, D.-W., Kim, J. J., Shin, J. W., Kim, J. H. \& Moon, D. (2015a). Acta Cryst. E71, 779-782.

Kim, D.-W., Shin, J. W. \& Moon, D. (2015b). Acta Cryst. E71, 136-138.

Lehn, J.-M. (2015). Angew. Chem. Int. Ed. 54, 3276-3289.

Melson, G. A. (1979). In Coordination Chemistry of Macrocyclic Compounds, 1st ed. New York: Plenum Press.

Min, K. S. \& Suh, M. P. (2001). Chem. Eur. J. 7, 303-313.

Otwinowski, Z. \& Minor, W. (1997). Methods in Enzymology, Vol. 276, Macromolecular Crystallography, Part A, edited by C. W. Carter Jr \& R. M. Sweet, pp. 307-326. New York: Academic Press.

Putz, H. \& Brandenburg, K. (2014). DIAMOND. Crystal Impact GbR, Bonn, Germany.

Ryoo, J. J., Shin, J. W., Dho, H.-S. \& Min, K. S. (2010). Inorg. Chem. 49, 7232-7234.

Sheldrick, G. M. (2015a). Acta Cryst. A71, 3-8.

Sheldrick, G. M. (2015b). Acta Cryst. C71, 3-8.

Shin, J. W., Eom, K. \& Moon, D. (2016a). J. Synchrotron Rad. 23, 369373.

Shin, J. W., Kim, D.-W., Kim, J. H. \& Moon, D. (2015). Acta Cryst. E71, 203-205.

Shin, J. W., Kim, D.-W. \& Moon, D. (2016b). Polyhedron, 105, 62-70.

Steed, J. W. \& Atwood, J. L. (2009). Supramol. Chem. 2nd ed. Chichester: John Wiley \& Sons Ltd.

Westrip, S. P. (2010). J. Appl. Cryst. 43, 920-925.

Xie, W.-P., Wang, N., Long, Y., Ran, X.-R., Gao, J.-Y., Chen, C.-J., Yue, S.-T. \& Cai, Y.-P. (2014). Inorg. Chem. Commun. 40, 151-156. 


\section{supporting information}

Acta Cryst. (2016). E72, 223-225 [doi:10.1107/S2056989016001031]

Crystal structure of trans-(1,8-dibutyl-1,3,6,8,10,13-hexaazacyclotetradecane$\left.\kappa^{4} N^{3}, N^{6}, N^{10}, N^{13}\right)$ bis(isonicotinato- $\kappa O$ ) nickel(II) determined from synchrotron data

Jong Won Shin, Dae-Woong Kim and Dohyun Moon

Computing details

Data collection: PAL BL2D-SMDC (Shin et al., 2016a); cell refinement: HKL-3000SM (Otwinowski \& Minor, 1997); data reduction: HKL-3000SM (Otwinowski \& Minor, 1997); program(s) used to solve structure: SHELXT (Sheldrick, 2015a); program(s) used to refine structure: SHELXL2014 (Sheldrick, 2015b); molecular graphics: DIAMOND (Putz \& Brandenburg, 2014); software used to prepare material for publication: publCIF (Westrip, 2010).

trans-(1,8-Dibutyl-1,3,6,8,10,13-hexaazacyclotetradecane- $\left.\kappa^{4} N^{3}, N^{6}, N^{10}, N^{13}\right)$ bis(isonicotinato- $\kappa O$ ) nickel(II)

Crystal data

$\left[\mathrm{Ni}\left(\mathrm{C}_{6} \mathrm{H}_{4} \mathrm{NO}_{2}\right)_{2}\left(\mathrm{C}_{16} \mathrm{H}_{38} \mathrm{~N}_{6}\right)\right]$

$M_{r}=617.44$

Triclinic, $P \overline{1}$

$a=8.0630(16) \AA$

$b=8.5110(17) \AA$

$c=10.927(2) \AA$

$\alpha=80.52(3)^{\circ}$

$\beta=88.26(3)^{\circ}$

$\gamma=86.44(3)^{\circ}$

$V=738.0(3) \AA^{3}$

\section{Data collection}

ADSC Q210 CCD area-detector diffractometer

Radiation source: PLSII 2D bending magnet $\omega$ scan

Absorption correction: empirical (using intensity measurements)

(HKL-3000SM SCALEPACK; Otwinowski \& Minor, 1997)

$T_{\min }=0.995, T_{\max }=0.998$

Refinement

Refinement on $F^{2}$

Least-squares matrix: full

$R\left[F^{2}>2 \sigma\left(F^{2}\right)\right]=0.040$

$w R\left(F^{2}\right)=0.110$

$S=1.04$

3879 reflections
$Z=1$

$F(000)=330$

$D_{\mathrm{x}}=1.389 \mathrm{Mg} \mathrm{m}^{-3}$

Synchrotron radiation, $\lambda=0.62998 \AA$

Cell parameters from 20128 reflections

$\theta=0.4-33.6^{\circ}$

$\mu=0.51 \mathrm{~mm}^{-1}$

$T=100 \mathrm{~K}$

Needle, pale pink

$0.01 \times 0.004 \times 0.004 \mathrm{~mm}$

7634 measured reflections

3879 independent reflections

3326 reflections with $I>2 \sigma(I)$

$R_{\text {int }}=0.023$

$\theta_{\max }=26.0^{\circ}, \theta_{\min }=2.5^{\circ}$

$h=-11 \rightarrow 11$

$k=-11 \rightarrow 11$

$l=-15 \rightarrow 15$

188 parameters

0 restraints

Hydrogen site location: inferred from neighbouring sites

$\mathrm{H}$-atom parameters constrained 
$w=1 /\left[\sigma^{2}\left(F_{\mathrm{o}}^{2}\right)+(0.0649 P)^{2}+0.1918 P\right]$

where $P=\left(F_{\mathrm{o}}^{2}+2 F_{\mathrm{c}}^{2}\right) / 3$

$(\Delta / \sigma)_{\max }<0.001$

$$
\Delta \rho_{\max }=1.12 \mathrm{e} \AA^{-3}
$$

$\Delta \rho_{\min }=-0.95$ e $\AA^{-3}$

\section{Special details}

Geometry. All e.s.d.'s (except the e.s.d. in the dihedral angle between two l.s. planes) are estimated using the full covariance matrix. The cell e.s.d.'s are taken into account individually in the estimation of e.s.d.'s in distances, angles and torsion angles; correlations between e.s.d.'s in cell parameters are only used when they are defined by crystal symmetry. An approximate (isotropic) treatment of cell e.s.d.'s is used for estimating e.s.d.'s involving 1.s. planes.

Fractional atomic coordinates and isotropic or equivalent isotropic displacement parameters $\left(\hat{A}^{2}\right)$

\begin{tabular}{|c|c|c|c|c|}
\hline & $x$ & $y$ & $z$ & $U_{\text {iso }} * / U_{\text {eq }}$ \\
\hline Nil & 0.5000 & 0.5000 & 0.5000 & $0.02066(10)$ \\
\hline O1 & $0.43513(15)$ & $0.41029(17)$ & $0.33736(11)$ & $0.0257(3)$ \\
\hline $\mathrm{O} 2$ & $0.18613(16)$ & 0.52908 (19) & $0.28022(12)$ & $0.0322(3)$ \\
\hline N1 & $0.27809(18)$ & $0.6311(2)$ & $0.50735(13)$ & 0.0244 \\
\hline H1 & 0.2171 & 0.6276 & 0.4296 & $0.029 *$ \\
\hline N2 & $0.61452(18)$ & 0.67953 (19) & $0.38249(13)$ & 0.0241 \\
\hline $\mathrm{H} 2$ & 0.5798 & 0.6766 & 0.2959 & $0.029 *$ \\
\hline N3 & $0.3946(2)$ & $0.8835(2)$ & $0.41256(14)$ & $0.0304(3)$ \\
\hline N4 & $0.3686(2)$ & $0.2837(2)$ & $-0.09149(14)$ & $0.0335(4)$ \\
\hline $\mathrm{C} 1$ & $0.1835(2)$ & $0.5457(3)$ & $0.61221(15)$ & $0.0276(4)$ \\
\hline $\mathrm{H} 1 \mathrm{~A}$ & 0.0642 & 0.5807 & 0.6057 & $0.033^{*}$ \\
\hline H1B & 0.2242 & 0.5692 & 0.6915 & $0.033^{*}$ \\
\hline $\mathrm{C} 2$ & $0.3004(2)$ & $0.8006(2)$ & $0.51521(16)$ & $0.0296(4)$ \\
\hline $\mathrm{H} 2 \mathrm{~A}$ & 0.1895 & 0.8566 & 0.5192 & $0.036^{*}$ \\
\hline $\mathrm{H} 2 \mathrm{~B}$ & 0.3574 & 0.8058 & 0.5933 & $0.036^{*}$ \\
\hline $\mathrm{C} 3$ & $0.5703(2)$ & $0.8414(2)$ & $0.41070(18)$ & $0.0308(4)$ \\
\hline $\mathrm{H} 3 \mathrm{~A}$ & 0.6153 & 0.8493 & 0.4926 & $0.037 *$ \\
\hline H3B & 0.6249 & 0.9202 & 0.3480 & $0.037 *$ \\
\hline $\mathrm{C} 4$ & $0.7938(2)$ & 0.6330 & $0.39084(16)$ & 0.0280 \\
\hline $\mathrm{H} 4 \mathrm{~A}$ & 0.8376 & 0.6572 & 0.4689 & $0.034^{*}$ \\
\hline H4B & 0.8552 & 0.6932 & 0.3203 & $0.034 *$ \\
\hline $\mathrm{C} 5$ & $0.3160(2)$ & $0.9034(2)$ & 0.29077 (17) & $0.0310(4)$ \\
\hline $\mathrm{H} 5 \mathrm{~A}$ & 0.2983 & 0.7968 & 0.2700 & $0.037^{*}$ \\
\hline H5B & 0.3922 & 0.9575 & 0.2269 & $0.037 *$ \\
\hline C6 & $0.1502(3)$ & $0.9999(3)$ & $0.28701(18)$ & $0.0341(4)$ \\
\hline H6A & 0.0727 & 0.9447 & 0.3493 & $0.041 *$ \\
\hline H6B & 0.1671 & 1.1058 & 0.3093 & $0.041 *$ \\
\hline $\mathrm{C} 7$ & 0.0730 & $1.0223(3)$ & 0.15954 (19) & $0.0390(5)$ \\
\hline H7A & 0.1527 & 1.0726 & 0.0966 & $0.047 *$ \\
\hline H7B & 0.0507 & 0.9166 & 0.1390 & $0.047 *$ \\
\hline $\mathrm{C} 8$ & $-0.0882(3)$ & $1.1257(4)$ & $0.1543(2)$ & $0.0502(6)$ \\
\hline $\mathrm{H} 8 \mathrm{~A}$ & -0.1692 & 1.0739 & 0.2140 & $0.075^{*}$ \\
\hline H8B & -0.1328 & 1.1395 & 0.0705 & $0.075^{*}$ \\
\hline $\mathrm{H} 8 \mathrm{C}$ & -0.0667 & 1.2302 & 0.1750 & $0.075^{*}$ \\
\hline C9 & $0.3163(2)$ & $0.4477(2)$ & $0.26295(15)$ & $0.0235(3)$ \\
\hline $\mathrm{C} 10$ & $0.4760(2)$ & 0.2978 & $0.10910(16)$ & $0.0294(4)$ \\
\hline
\end{tabular}




\begin{tabular}{lllll}
$\mathrm{H} 10$ & 0.5631 & 0.2691 & 0.1666 & $0.035^{*}$ \\
$\mathrm{C} 11$ & $0.3367(2)$ & $0.3884(2)$ & $0.13963(14)$ & $0.0234(3)$ \\
$\mathrm{C} 12$ & $0.2134(2)$ & $0.4252(3)$ & $0.05120(16)$ & $0.0300(4)$ \\
$\mathrm{H} 12$ & 0.1158 & 0.4874 & 0.0676 & $0.036^{*}$ \\
$\mathrm{C} 13$ & $0.2347(2)$ & $0.3703(3)$ & $-0.06044(17)$ & $0.0339(4)$ \\
$\mathrm{H} 13$ & 0.1486 & 0.3957 & -0.1191 & $0.041^{*}$ \\
$\mathrm{C} 14$ & $0.4868(2)$ & $0.2496(3)$ & $-0.00601(17)$ & $0.0340(4)$ \\
$\mathrm{H} 14$ & 0.5838 & 0.1887 & -0.0256 & $0.041^{*}$ \\
\hline
\end{tabular}

Atomic displacement parameters $\left(\AA^{2}\right)$

\begin{tabular}{lllllll}
\hline & $U^{11}$ & $U^{22}$ & $U^{33}$ & $U^{12}$ & $U^{13}$ & $U^{23}$ \\
\hline Ni1 & $0.01767(15)$ & $0.03464(19)$ & $0.00987(14)$ & $0.00142(11)$ & $-0.00201(9)$ & $-0.00504(11)$ \\
O1 & $0.0246(6)$ & $0.0412(7)$ & $0.0122(5)$ & $0.0012(5)$ & $-0.0054(4)$ & $-0.0073(5)$ \\
O2 & $0.0235(6)$ & $0.0543(9)$ & $0.0207(6)$ & $0.0047(6)$ & $-0.0043(5)$ & $-0.0139(6)$ \\
N1 & $0.0225(7)$ & $0.0384(8)$ & $0.0122(6)$ & $0.0027(6)$ & $-0.0022(5)$ & $-0.0048(6)$ \\
N2 & $0.0227(7)$ & $0.0355(8)$ & $0.0142(6)$ & $0.0007(6)$ & $-0.0016(5)$ & $-0.0049(6)$ \\
N3 & $0.0338(8)$ & $0.0354(9)$ & $0.0209(7)$ & $0.0055(7)$ & $-0.0018(6)$ & $-0.0041(7)$ \\
N4 & $0.0344(8)$ & $0.0525(11)$ & $0.0148(6)$ & $0.0002(7)$ & $-0.0027(6)$ & $-0.0091(7)$ \\
C1 & $0.0186(7)$ & $0.0485(11)$ & $0.0149(7)$ & $0.0026(7)$ & $0.0012(5)$ & $-0.0052(7)$ \\
C2 & $0.0331(9)$ & $0.0384(10)$ & $0.0169(8)$ & $0.0078(8)$ & $-0.0008(6)$ & $-0.0068(7)$ \\
C3 & $0.0339(9)$ & $0.0350(10)$ & $0.0240(8)$ & $-0.0011(7)$ & $-0.0018(7)$ & $-0.0063(8)$ \\
C4 & $0.0205(8)$ & $0.0460(11)$ & $0.0173(7)$ & $-0.0038(7)$ & $0.0002(6)$ & $-0.0043(7)$ \\
C5 & $0.0376(10)$ & $0.0345(10)$ & $0.0188(8)$ & $0.0059(8)$ & $-0.0014(7)$ & $-0.0011(7)$ \\
C6 & $0.0356(10)$ & $0.0414(11)$ & $0.0227(9)$ & $0.0064(8)$ & $-0.0008(7)$ & $-0.0009(8)$ \\
C7 & $0.0394(11)$ & $0.0506(13)$ & $0.0243(9)$ & $0.0048(9)$ & $-0.0033(7)$ & $-0.0006(9)$ \\
C8 & $0.0400(12)$ & $0.0733(18)$ & $0.0320(11)$ & $0.0119(11)$ & $-0.0033(9)$ & $0.0018(11)$ \\
C9 & $0.0213(7)$ & $0.0366(9)$ & $0.0128(7)$ & $-0.0044(6)$ & $-0.0018(5)$ & $-0.0040(6)$ \\
C10 & $0.0258(8)$ & $0.0468(11)$ & $0.0160(7)$ & $0.0028(7)$ & $-0.0044(6)$ & $-0.0071(7)$ \\
C11 & $0.0222(7)$ & $0.0365(9)$ & $0.0119(7)$ & $-0.0038(7)$ & $-0.0016(5)$ & $-0.0047(7)$ \\
C12 & $0.0257(8)$ & $0.0484(11)$ & $0.0158(7)$ & $0.0020(8)$ & $-0.0044(6)$ & $-0.0062(8)$ \\
C13 & $0.0307(9)$ & $0.0562(13)$ & $0.0157(8)$ & $0.0009(8)$ & $-0.0073(6)$ & $-0.0085(8)$ \\
C14 & $0.0306(9)$ & $0.0534(12)$ & $0.0186(8)$ & $0.0056(8)$ & $-0.0017(7)$ & $-0.0106(8)$ \\
& & & & & & \\
\hline & & & & & &
\end{tabular}

Geometric parameters $\left(\AA,^{\circ}\right)$

\begin{tabular}{llll}
\hline $\mathrm{Ni} 1-\mathrm{N} 1^{\mathrm{i}}$ & $2.0559(16)$ & $\mathrm{C} 3-\mathrm{H} 3 \mathrm{~B}$ & 0.9900 \\
$\mathrm{Ni} 1-\mathrm{N} 1$ & $2.0559(16)$ & $\mathrm{C} 4-\mathrm{C} 1^{\mathrm{i}}$ & $1.526(3)$ \\
$\mathrm{Ni} 1-\mathrm{N} 2$ & $2.0720(17)$ & $\mathrm{C} 4-\mathrm{H} 4 \mathrm{~A}$ & 0.9900 \\
$\mathrm{~N} 1-\mathrm{N} 2^{\mathrm{i}}$ & $2.0720(17)$ & $\mathrm{C} 4-\mathrm{H} 4 \mathrm{~B}$ & 0.9900 \\
$\mathrm{Ni1}-\mathrm{O} 1^{\mathrm{i}}$ & $2.1371(13)$ & $\mathrm{C} 5-\mathrm{C} 6$ & $1.523(3)$ \\
$\mathrm{Ni1}-\mathrm{O} 1$ & $2.1372(13)$ & $\mathrm{C} 5-\mathrm{H} 5 \mathrm{~A}$ & 0.9900 \\
$\mathrm{O} 1-\mathrm{C} 9$ & $1.2669(19)$ & $\mathrm{C} 5-\mathrm{H} 5 \mathrm{~B}$ & 0.9900 \\
$\mathrm{O} 2-\mathrm{C} 9$ & $1.248(2)$ & $\mathrm{C} 6-\mathrm{C} 7$ & $1.521(3)$ \\
$\mathrm{N} 1-\mathrm{C} 1$ & $1.471(2)$ & $\mathrm{C} 6-\mathrm{H} 6 \mathrm{~A}$ & 0.9900 \\
$\mathrm{~N} 1-\mathrm{C} 2$ & $1.481(3)$ & $\mathrm{C} 6-\mathrm{H} 6 \mathrm{~B}$ & 0.9900 \\
$\mathrm{~N} 1-\mathrm{H} 1$ & 1.0000 & $\mathrm{C} 7-\mathrm{C} 8$ & $1.521(3)$ \\
$\mathrm{N} 2-\mathrm{C} 4$ & $1.477(2)$ & $\mathrm{C} 7-\mathrm{H} 7 \mathrm{~A}$ & 0.9900
\end{tabular}




\begin{tabular}{|c|c|c|c|}
\hline $\mathrm{N} 2-\mathrm{C} 3$ & $1.481(3)$ & $\mathrm{C} 7-\mathrm{H} 7 \mathrm{~B}$ & 0.9900 \\
\hline $\mathrm{N} 2-\mathrm{H} 2$ & 1.0000 & $\mathrm{C} 8-\mathrm{H} 8 \mathrm{~A}$ & 0.9800 \\
\hline $\mathrm{N} 3-\mathrm{C} 3$ & $1.440(3)$ & $\mathrm{C} 8-\mathrm{H} 8 \mathrm{~B}$ & 0.9800 \\
\hline $\mathrm{N} 3-\mathrm{C} 2$ & $1.444(2)$ & $\mathrm{C} 8-\mathrm{H} 8 \mathrm{C}$ & 0.9800 \\
\hline $\mathrm{N} 3-\mathrm{C} 5$ & $1.471(2)$ & $\mathrm{C} 9-\mathrm{C} 11$ & $1.516(2)$ \\
\hline $\mathrm{N} 4-\mathrm{C} 13$ & $1.336(3)$ & $\mathrm{C} 10-\mathrm{C} 14$ & $1.384(3)$ \\
\hline $\mathrm{N} 4-\mathrm{C} 14$ & $1.340(2)$ & $\mathrm{C} 10-\mathrm{C} 11$ & $1.386(3)$ \\
\hline $\mathrm{C} 1-\mathrm{C} 4^{\mathrm{i}}$ & $1.526(3)$ & $\mathrm{C} 10-\mathrm{H} 10$ & 0.9500 \\
\hline $\mathrm{C} 1-\mathrm{H} 1 \mathrm{~A}$ & 0.9900 & $\mathrm{C} 11-\mathrm{C} 12$ & $1.393(2)$ \\
\hline $\mathrm{C} 1-\mathrm{H} 1 \mathrm{~B}$ & 0.9900 & $\mathrm{C} 12-\mathrm{C} 13$ & $1.379(3)$ \\
\hline $\mathrm{C} 2-\mathrm{H} 2 \mathrm{~A}$ & 0.9900 & $\mathrm{C} 12-\mathrm{H} 12$ & 0.9500 \\
\hline $\mathrm{C} 2-\mathrm{H} 2 \mathrm{~B}$ & 0.9900 & $\mathrm{C} 13-\mathrm{H} 13$ & 0.9500 \\
\hline $\mathrm{C} 3-\mathrm{H} 3 \mathrm{~A}$ & 0.9900 & $\mathrm{C} 14-\mathrm{H} 14$ & 0.9500 \\
\hline $\mathrm{N} 1{ }^{\mathrm{i}}-\mathrm{Ni} 1-\mathrm{N} 1$ & 180.0 & $\mathrm{H} 3 \mathrm{~A}-\mathrm{C} 3-\mathrm{H} 3 \mathrm{~B}$ & 107.6 \\
\hline $\mathrm{N} 1{ }^{\mathrm{i}}-\mathrm{Ni} 1-\mathrm{N} 2$ & $85.97(6)$ & $\mathrm{N} 2-\mathrm{C} 4-\mathrm{C} 1^{\mathrm{i}}$ & $108.14(15)$ \\
\hline $\mathrm{N} 1-\mathrm{Ni1}-\mathrm{N} 2$ & $94.03(6)$ & $\mathrm{N} 2-\mathrm{C} 4-\mathrm{H} 4 \mathrm{~A}$ & 110.1 \\
\hline $\mathrm{N} 1^{\mathrm{i}}-\mathrm{N} i 1-\mathrm{N} 2^{\mathrm{i}}$ & $94.03(6)$ & $\mathrm{C}^{\mathrm{i}}-\mathrm{C} 4-\mathrm{H} 4 \mathrm{~A}$ & 110.1 \\
\hline $\mathrm{N} 1-\mathrm{N} i 1-\mathrm{N} 2^{\mathrm{i}}$ & $85.97(6)$ & $\mathrm{N} 2-\mathrm{C} 4-\mathrm{H} 4 \mathrm{~B}$ & 110.1 \\
\hline $\mathrm{N} 2-\mathrm{Ni} 1-\mathrm{N} 2^{\mathrm{i}}$ & 180.0 & $\mathrm{C} 1-\mathrm{C} 4-\mathrm{H} 4 \mathrm{~B}$ & 110.1 \\
\hline $\mathrm{N} 1{ }^{\mathrm{i}}-\mathrm{Ni} 1-\mathrm{O} 1^{\mathrm{i}}$ & $93.29(6)$ & $\mathrm{H} 4 \mathrm{~A}-\mathrm{C} 4-\mathrm{H} 4 \mathrm{~B}$ & 108.4 \\
\hline $\mathrm{N} 1-\mathrm{Ni} 1-\mathrm{O} 1^{\mathrm{i}}$ & $86.71(6)$ & $\mathrm{N} 3-\mathrm{C} 5-\mathrm{C} 6$ & $112.79(16)$ \\
\hline $\mathrm{N} 2-\mathrm{Ni} 1-\mathrm{O} 1^{\mathrm{i}}$ & $92.90(6)$ & N3-C5-H5A & 109.0 \\
\hline $\mathrm{N} 22^{\mathrm{i}}-\mathrm{Ni} 1-\mathrm{O} 1^{\mathrm{i}}$ & $87.10(6)$ & $\mathrm{C} 6-\mathrm{C} 5-\mathrm{H} 5 \mathrm{~A}$ & 109.0 \\
\hline $\mathrm{N} 1{ }^{\mathrm{i}}-\mathrm{Ni} 1-\mathrm{O} 1$ & $86.71(6)$ & N3- $55-\mathrm{H} 5 \mathrm{~B}$ & 109.0 \\
\hline $\mathrm{N} 1-\mathrm{Ni} 1-\mathrm{O} 1$ & $93.29(6)$ & $\mathrm{C} 6-\mathrm{C} 5-\mathrm{H} 5 \mathrm{~B}$ & 109.0 \\
\hline $\mathrm{N} 2-\mathrm{Ni} 1-\mathrm{O} 1$ & $87.10(6)$ & $\mathrm{H} 5 \mathrm{~A}-\mathrm{C} 5-\mathrm{H} 5 \mathrm{~B}$ & 107.8 \\
\hline $\mathrm{N} 2 \mathrm{i}-\mathrm{Ni} 1-\mathrm{O} 1$ & $92.90(6)$ & $\mathrm{C} 7-\mathrm{C} 6-\mathrm{C} 5$ & $111.93(17)$ \\
\hline $\mathrm{O} 1{ }^{\mathrm{i}}-\mathrm{Ni1}-\mathrm{O} 1$ & 180.0 & $\mathrm{C} 7-\mathrm{C} 6-\mathrm{H} 6 \mathrm{~A}$ & 109.2 \\
\hline $\mathrm{C} 9-\mathrm{O} 1-\mathrm{Ni1}$ & $131.99(12)$ & $\mathrm{C} 5-\mathrm{C} 6-\mathrm{H} 6 \mathrm{~A}$ & 109.2 \\
\hline $\mathrm{C} 1-\mathrm{N} 1-\mathrm{C} 2$ & 114.34 (14) & $\mathrm{C} 7-\mathrm{C} 6-\mathrm{H} 6 \mathrm{~B}$ & 109.2 \\
\hline $\mathrm{C} 1-\mathrm{N} 1-\mathrm{Ni} 1$ & $105.52(11)$ & $\mathrm{C} 5-\mathrm{C} 6-\mathrm{H} 6 \mathrm{~B}$ & 109.2 \\
\hline $\mathrm{C} 2-\mathrm{N} 1-\mathrm{Ni} 1$ & $112.75(11)$ & $\mathrm{H} 6 \mathrm{~A}-\mathrm{C} 6-\mathrm{H} 6 \mathrm{~B}$ & 107.9 \\
\hline $\mathrm{C} 1-\mathrm{N} 1-\mathrm{H} 1$ & 108.0 & $\mathrm{C} 8-\mathrm{C} 7-\mathrm{C} 6$ & $111.81(19)$ \\
\hline $\mathrm{C} 2-\mathrm{N} 1-\mathrm{H} 1$ & 108.0 & $\mathrm{C} 8-\mathrm{C} 7-\mathrm{H} 7 \mathrm{~A}$ & 109.3 \\
\hline $\mathrm{Ni} 1-\mathrm{N} 1-\mathrm{H} 1$ & 108.0 & $\mathrm{C} 6-\mathrm{C} 7-\mathrm{H} 7 \mathrm{~A}$ & 109.3 \\
\hline $\mathrm{C} 4-\mathrm{N} 2-\mathrm{C} 3$ & $113.96(15)$ & $\mathrm{C} 8-\mathrm{C} 7-\mathrm{H} 7 \mathrm{~B}$ & 109.3 \\
\hline $\mathrm{C} 4-\mathrm{N} 2-\mathrm{Ni} 1$ & $104.76(11)$ & $\mathrm{C} 6-\mathrm{C} 7-\mathrm{H} 7 \mathrm{~B}$ & 109.3 \\
\hline $\mathrm{C} 3-\mathrm{N} 2-\mathrm{Ni} 1$ & $113.72(11)$ & $\mathrm{H} 7 \mathrm{~A}-\mathrm{C} 7-\mathrm{H} 7 \mathrm{~B}$ & 107.9 \\
\hline $\mathrm{C} 4-\mathrm{N} 2-\mathrm{H} 2$ & 108.0 & $\mathrm{C} 7-\mathrm{C} 8-\mathrm{H} 8 \mathrm{~A}$ & 109.5 \\
\hline $\mathrm{C} 3-\mathrm{N} 2-\mathrm{H} 2$ & 108.0 & $\mathrm{C} 7-\mathrm{C} 8-\mathrm{H} 8 \mathrm{~B}$ & 109.5 \\
\hline $\mathrm{Ni} 1-\mathrm{N} 2-\mathrm{H} 2$ & 108.0 & $\mathrm{H} 8 \mathrm{~A}-\mathrm{C} 8-\mathrm{H} 8 \mathrm{~B}$ & 109.5 \\
\hline $\mathrm{C} 3-\mathrm{N} 3-\mathrm{C} 2$ & $115.84(15)$ & $\mathrm{C} 7-\mathrm{C} 8-\mathrm{H} 8 \mathrm{C}$ & 109.5 \\
\hline $\mathrm{C} 3-\mathrm{N} 3-\mathrm{C} 5$ & $114.58(15)$ & $\mathrm{H} 8 \mathrm{~A}-\mathrm{C} 8-\mathrm{H} 8 \mathrm{C}$ & 109.5 \\
\hline $\mathrm{C} 2-\mathrm{N} 3-\mathrm{C} 5$ & $115.55(16)$ & $\mathrm{H} 8 \mathrm{~B}-\mathrm{C} 8-\mathrm{H} 8 \mathrm{C}$ & 109.5 \\
\hline $\mathrm{C} 13-\mathrm{N} 4-\mathrm{C} 14$ & $116.05(17)$ & $\mathrm{O} 2-\mathrm{C} 9-\mathrm{O} 1$ & $126.88(16)$ \\
\hline $\mathrm{N} 1-\mathrm{C} 1-\mathrm{C}^{\mathrm{i}}$ & $108.60(14)$ & $\mathrm{O} 2-\mathrm{C} 9-\mathrm{C} 11$ & $117.12(15)$ \\
\hline $\mathrm{N} 1-\mathrm{C} 1-\mathrm{H} 1 \mathrm{~A}$ & 110.0 & $\mathrm{O} 1-\mathrm{C} 9-\mathrm{C} 11$ & $115.99(16)$ \\
\hline
\end{tabular}




\begin{tabular}{|c|c|c|c|}
\hline $\mathrm{C} 4-\mathrm{C} 1-\mathrm{H} 1 \mathrm{~A}$ & 110.0 & $\mathrm{C} 14-\mathrm{C} 10-\mathrm{C} 11$ & $119.30(17)$ \\
\hline $\mathrm{N} 1-\mathrm{C} 1-\mathrm{H} 1 \mathrm{~B}$ & 110.0 & $\mathrm{C} 14-\mathrm{C} 10-\mathrm{H} 10$ & 120.4 \\
\hline $\mathrm{C} 4-\mathrm{C} 1-\mathrm{H} 1 \mathrm{~B}$ & 110.0 & $\mathrm{C} 11-\mathrm{C} 10-\mathrm{H} 10$ & 120.4 \\
\hline $\mathrm{H} 1 \mathrm{~A}-\mathrm{C} 1-\mathrm{H} 1 \mathrm{~B}$ & 108.4 & $\mathrm{C} 10-\mathrm{C} 11-\mathrm{C} 12$ & $117.25(16)$ \\
\hline $\mathrm{N} 3-\mathrm{C} 2-\mathrm{N} 1$ & $114.07(15)$ & $\mathrm{C} 10-\mathrm{C} 11-\mathrm{C} 9$ & $122.61(15)$ \\
\hline $\mathrm{N} 3-\mathrm{C} 2-\mathrm{H} 2 \mathrm{~A}$ & 108.7 & $\mathrm{C} 12-\mathrm{C} 11-\mathrm{C} 9$ & $120.14(16)$ \\
\hline $\mathrm{N} 1-\mathrm{C} 2-\mathrm{H} 2 \mathrm{~A}$ & 108.7 & $\mathrm{C} 13-\mathrm{C} 12-\mathrm{C} 11$ & $119.15(18)$ \\
\hline $\mathrm{N} 3-\mathrm{C} 2-\mathrm{H} 2 \mathrm{~B}$ & 108.7 & $\mathrm{C} 13-\mathrm{C} 12-\mathrm{H} 12$ & 120.4 \\
\hline $\mathrm{N} 1-\mathrm{C} 2-\mathrm{H} 2 \mathrm{~B}$ & 108.7 & $\mathrm{C} 11-\mathrm{C} 12-\mathrm{H} 12$ & 120.4 \\
\hline $\mathrm{H} 2 \mathrm{~A}-\mathrm{C} 2-\mathrm{H} 2 \mathrm{~B}$ & 107.6 & $\mathrm{~N} 4-\mathrm{C} 13-\mathrm{C} 12$ & $124.30(17)$ \\
\hline $\mathrm{N} 3-\mathrm{C} 3-\mathrm{N} 2$ & $114.51(16)$ & $\mathrm{N} 4-\mathrm{C} 13-\mathrm{H} 13$ & 117.8 \\
\hline $\mathrm{N} 3-\mathrm{C} 3-\mathrm{H} 3 \mathrm{~A}$ & 108.6 & $\mathrm{C} 12-\mathrm{C} 13-\mathrm{H} 13$ & 117.8 \\
\hline $\mathrm{N} 2-\mathrm{C} 3-\mathrm{H} 3 \mathrm{~A}$ & 108.6 & $\mathrm{~N} 4-\mathrm{C} 14-\mathrm{C} 10$ & $123.94(18)$ \\
\hline $\mathrm{N} 3-\mathrm{C} 3-\mathrm{H} 3 \mathrm{~B}$ & 108.6 & N4-C14-H14 & 118.0 \\
\hline $\mathrm{N} 2-\mathrm{C} 3-\mathrm{H} 3 \mathrm{~B}$ & 108.6 & $\mathrm{C} 10-\mathrm{C} 14-\mathrm{H} 14$ & 118.0 \\
\hline $\mathrm{C} 2-\mathrm{N} 1-\mathrm{C} 1-\mathrm{C} 4^{\mathrm{i}}$ & $-166.23(14)$ & $\mathrm{C} 5-\mathrm{C} 6-\mathrm{C} 7-\mathrm{C} 8$ & $-177.2(2)$ \\
\hline $\mathrm{Ni} 1-\mathrm{N} 1-\mathrm{C} 1-\mathrm{C} 4^{\mathrm{i}}$ & $-41.74(15)$ & $\mathrm{Ni1}-\mathrm{O} 1-\mathrm{C} 9-\mathrm{O} 2$ & $-15.1(3)$ \\
\hline $\mathrm{C} 3-\mathrm{N} 3-\mathrm{C} 2-\mathrm{N} 1$ & $-72.6(2)$ & $\mathrm{Ni1}-\mathrm{O} 1-\mathrm{C} 9-\mathrm{C} 11$ & $164.15(12)$ \\
\hline $\mathrm{C} 5-\mathrm{N} 3-\mathrm{C} 2-\mathrm{N} 1$ & $65.5(2)$ & $\mathrm{C} 14-\mathrm{C} 10-\mathrm{C} 11-\mathrm{C} 12$ & $0.4(3)$ \\
\hline $\mathrm{C} 1-\mathrm{N} 1-\mathrm{C} 2-\mathrm{N} 3$ & $179.49(14)$ & $\mathrm{C} 14-\mathrm{C} 10-\mathrm{C} 11-\mathrm{C} 9$ & $-179.18(18)$ \\
\hline $\mathrm{Ni} 1-\mathrm{N} 1-\mathrm{C} 2-\mathrm{N} 3$ & $58.94(17)$ & $\mathrm{O} 2-\mathrm{C} 9-\mathrm{C} 11-\mathrm{C} 10$ & $179.64(18)$ \\
\hline $\mathrm{C} 2-\mathrm{N} 3-\mathrm{C} 3-\mathrm{N} 2$ & $70.3(2)$ & $\mathrm{O} 1-\mathrm{C} 9-\mathrm{C} 11-\mathrm{C} 10$ & $0.3(3)$ \\
\hline $\mathrm{C} 5-\mathrm{N} 3-\mathrm{C} 3-\mathrm{N} 2$ & $-68.2(2)$ & $\mathrm{O} 2-\mathrm{C} 9-\mathrm{C} 11-\mathrm{C} 12$ & $0.1(3)$ \\
\hline $\mathrm{C} 4-\mathrm{N} 2-\mathrm{C} 3-\mathrm{N} 3$ & $-175.30(14)$ & $\mathrm{O} 1-\mathrm{C} 9-\mathrm{C} 11-\mathrm{C} 12$ & $-179.24(17)$ \\
\hline $\mathrm{Ni} 1-\mathrm{N} 2-\mathrm{C} 3-\mathrm{N} 3$ & $-55.32(18)$ & $\mathrm{C} 10-\mathrm{C} 11-\mathrm{C} 12-\mathrm{C} 13$ & $0.3(3)$ \\
\hline $\mathrm{C} 3-\mathrm{N} 2-\mathrm{C} 4-\mathrm{C}^{\mathrm{i}}$ & $167.84(13)$ & $\mathrm{C} 9-\mathrm{C} 11-\mathrm{C} 12-\mathrm{C} 13$ & $179.86(18)$ \\
\hline $\mathrm{Ni} 1-\mathrm{N} 2-\mathrm{C} 4-\mathrm{C} 1^{\mathrm{i}}$ & $42.93(14)$ & $\mathrm{C} 14-\mathrm{N} 4-\mathrm{C} 13-\mathrm{C} 12$ & $0.4(3)$ \\
\hline $\mathrm{C} 3-\mathrm{N} 3-\mathrm{C} 5-\mathrm{C} 6$ & $-160.49(18)$ & $\mathrm{C} 11-\mathrm{C} 12-\mathrm{C} 13-\mathrm{N} 4$ & $-0.7(3)$ \\
\hline $\mathrm{C} 2-\mathrm{N} 3-\mathrm{C} 5-\mathrm{C} 6$ & $60.9(2)$ & $\mathrm{C} 13-\mathrm{N} 4-\mathrm{C} 14-\mathrm{C} 10$ & $0.4(3)$ \\
\hline $\mathrm{N} 3-\mathrm{C} 5-\mathrm{C} 6-\mathrm{C} 7$ & $178.71(18)$ & $\mathrm{C} 11-\mathrm{C} 10-\mathrm{C} 14-\mathrm{N} 4$ & $-0.8(3)$ \\
\hline
\end{tabular}

Symmetry code: (i) $-x+1,-y+1,-z+1$.

Hydrogen-bond geometry $\left(A,{ }^{\circ}\right)$

\begin{tabular}{lllll}
\hline$D-\mathrm{H} \cdots A$ & $D-\mathrm{H}$ & $\mathrm{H} \cdots A$ & $D \cdots A$ & $D-\mathrm{H} \cdots A$ \\
\hline $\mathrm{N} 1-\mathrm{H} 1 \cdots \mathrm{O} 2$ & 1.00 & 1.98 & $2.892(2)$ & 150 \\
$\mathrm{~N} 2-\mathrm{H} 2 \cdots \mathrm{N} 4{ }^{\text {ii }}$ & 1.00 & 2.23 & $3.143(2)$ & 151 \\
\hline
\end{tabular}

Symmetry code: (ii) $-x+1,-y+1,-z$. 